\title{
Fabrication of alginate-gelatin crosslinked hydrogel microcapsules and evaluation of the microstructure and physico-chemical properties
}

Cite this: J. Mater. Chem. B, 2014, 2 , 1470

Received 25th October 2013

Accepted 9th December 2013

DOI: 10.1039/c3tb21509a

www.rsc.org/MaterialsB

\author{
Bapi Sarker, ${ }^{a}$ Dimitrios G. Papageorgiou, ${ }^{\text {b }}$ Raquel Silva, ${ }^{a}$ Tobias Zehnder, ${ }^{a}$ \\ Farhana Gul-E-Noor, $\dagger^{\mathrm{c}}$ Marko Bertmer, ${ }^{\mathrm{c}}$ Joachim Kaschta, ${ }^{\mathrm{d}}$ Konstantinos Chrissafis, ${ }^{\mathrm{b}}$ \\ Rainer Detsch ${ }^{a}$ and Aldo R. Boccaccini ${ }^{\star a}$
}

Microencapsulation of cells by using biodegradable hydrogels offers numerous attractive features for a variety of biomedical applications including tissue engineering. This study highlights the fabrication of microcapsules from an alginate-gelatin crosslinked hydrogel (ADA-GEL) and presents the evaluation of the physico-chemical properties of the new microcapsules which are relevant for designing suitable microcapsules for tissue engineering. Alginate di-aldehyde (ADA) was synthesized by periodate oxidation of alginate which facilitates crosslinking with gelatin through Schiff's base formation between the free amino groups of gelatin and the available aldehyde groups of ADA. Formation of Schiff's base in ADAGEL and aldehyde groups in ADA was confirmed by FTIR and NMR spectroscopy, respectively. Thermal degradation behavior of films and microcapsules fabricated from alginate, ADA and ADA-GEL was dependent on the hydrogel composition. The gelation time of ADA-GEL was found to decrease with increasing gelatin content. The swelling ratio of ADA-GEL microcapsules of all compositions was significantly decreased, whereas the degradability was found to increase with the increase of gelatin ratio. The surface morphology of the ADA-GEL microcapsules was totally different from that of alginate and ADA microcapsules, observed by SEM. Two different buffer solutions (with and without calcium salt) have an influence on the stability of microcapsules which had a significant effect on the gelatin release profile of ADA-GEL microcapsules in these two buffer solutions.

\section{Introduction}

Hydrogels, from naturally occurring biopolymers, are an important class of biomaterials that are widely used in the pharmaceutical and biomedical sectors. ${ }^{1,2}$ Among the naturally occurring biopolymers, alginate and gelatin are extensively used for many biomedical applications because of their biocompatibility and biodegradability.,

Alginate is commercially available as a sodium salt of alginic acid which is a polysaccharide and composed of $\beta$-D-mannuronic acid and $\alpha$-L-guluronic acid units arranged in blocks of poly(mannuronate) and poly(guluronate). ${ }^{\mathbf{1 , 4}}$ Alginate is widely

anstitute of Biomaterials, Department of Materials Science and Engineering, University of Erlangen-Nuremberg, Cauerstrasse 6, 91058 Erlangen, Germany. E-mail: aldo.boccaccini@ww.uni-erlangen.de

${ }^{b}$ Solid State Physics Section, Physics Department, Aristotle University of Thessaloniki, 54124 Thessaloniki, Greece

'Institute of Experimental Physics II, University of Leipzig, Linnéstr. 5, D-04103 Leipzig, Germany

${ }^{d}$ Institute of Polymer Materials, University of Erlangen-Nuremberg, Martensstrasse 7, D-91058 Erlangen, Germany

$\dagger$ Current address: Department of Chemistry, University of Western Ontario, London, Ontario, N6A5B7, Canada. used in cell encapsulation ${ }^{4-7}$ and biofabrication ${ }^{8-12}$ because of its rapid ionic gelation with divalent cations which is achieved through the poly(guluronate) portion. ${ }^{\mathbf{1 3 , 1 4}}$ However, usually alginate does not promote efficient cell attachment leading to very poor cell-material interactions, in addition alginate possesses very slow degradability and uncontrolled degradation kinetics. ${ }^{14-17}$ These limitations of alginate can be overcome by incorporation of gelatin through covalent crosslinking with alginate di-aldehyde (ADA) ${ }^{18,19}$ ADA is a partially oxidized product of alginate which facilitates the covalent crosslinking with gelatin through Schiff's base formation due to the reaction of free amino groups of lysine or hydroxylysine amino acid residues of gelatin and available aldehyde groups of ADA. ${ }^{3,18}$ Moreover, the biodegradability of the alginate-gelatin crosslinked hydrogel can be tuned by using ADA of different degrees of oxidation which can control the hydrolysis properties of alginate $^{\mathbf{2 0 , 2 1}}$ and also by changing the composition of ADA and gelatin.

Gelatin is a biodegradable protein, produced by acidic or basic hydrolysis of collagen, which involves breaking of the collagen's triple helix structure into random coils. ${ }^{22}$ The sol state of gelatin transforms into the gel state upon cooling of aqueous solution of gelatin, which involves a partial 
rearrangement of the gelatin structure from random coil into triple helix. ${ }^{23-25}$ Gelatin is widely used in biomedical applications, for example in tissue engineering, wound dressing, gene therapy, and drug delivery due to its high biocompatibility and biodegradability. ${ }^{26}$ However, gelatin's poor mechanical properties, which limit its wider possible applications, can be overcome by covalent crosslinking with ADA. ${ }^{3}$

Therefore the main goal of this investigation was the synthesis of the alginate-gelatin crosslinked hydrogel (ADAGEL) aiming to fabricate microcapsules. To the best of our knowledge, this is the first study reporting the fabrication and characterization of microcapsules from ADA-GEL. Microencapsulation is a promising technology in tissue engineering that has drawn interest in recent years. This technique is widely used for encapsulation of cells or bioactive molecules, drug delivery, and gene therapy. ${ }^{27}$ Hydrogels are the most widely used materials for cell microencapsulation because of their high porosity that leads to high permeability of oxygen, nutrients, and metabolites. At the same time hydrogels can protect the encapsulated cells from the host immune system and high molecular weight complexes like immunoglobulin., ${ }^{5,28}$ Moreover, the soft and flexible nature of hydrogels reduces the mechanical stress and friction on encapsulated cells and also on adjacent tissue upon transplantation. ${ }^{4}$ Most importantly, hydrogels are highly hydrophilic which leads to high water content, providing a tissue like environment to the encapsulated cells. ${ }^{29}$

On the basis of these advantages, it is expected that the novel microcapsules from ADA-GEL can offer possibilities for future studies in tissue engineering. Indeed ADA-GEL is already known as a favorable material for tissue engineering applications..$^{18,19,30}$ However, the material has been used mainly for 2-D cell culture studies. In the present investigation, we were able to fabricate microcapsules from ADA-GEL by controlling the synthesis process of ADA-GEL, which is helpful for cell studies in 3-D environments by enabling cell encapsulation.

\section{Experimental section}

\section{Materials}

Sodium alginate (sodium salt of alginic acid from brown algae, suitable for immobilization of micro-organisms, MW 100 000$200000 \mathrm{~g} \mathrm{~mol}^{-1}$, guluronic acid content $65-70 \%$ ) and gelatin (Bloom 300, Type A, porcine skin, suitable for cell culture) were obtained from Sigma-Aldrich, Germany. Sodium metaperiodate and calcium chloride di-hydrate $\left(\mathrm{CaCl}_{2} \cdot 2 \mathrm{H}_{2} \mathrm{O}\right)$ were purchased from VWR international, Belgium.

\section{Oxidation reaction on sodium alginate}

ADA was synthesized by controlled oxidation of sodium alginate in an equal volume of ethanol-water mixture to facilitate crosslinking of sodium alginate with gelatin. ${ }^{3,21,31,32}$ Briefly, $5 \mathrm{~g}$ of alginate were dispersed in $25 \mathrm{ml}$ ethanol (VWR int., Belgium) and $1.605 \mathrm{~g}$ of sodium metaperiodate were dissolved in $25 \mathrm{ml}$ deionized (DI) water to get $7.5 \mathrm{mmol}$ periodate solution. The periodate solution was slowly added to the sodium alginate dispersion, which was continuously stirred under dark conditions at room temperature. The reaction was quenched after 6 hours by adding $5 \mathrm{ml}$ of ethylene glycol (relative density 1.115) (VWR int., Belgium) under continuous stirring for 30 minutes. The resultant suspension was dialyzed against ultrapure water (Direct-Q®, Merck Millipore, Germany) using a dialysis membrane (MWCO: 6000-8000 Da, Spectrum Lab, USA) for 7 days with several changes of water until the dialysate was periodate free. The absence of periodate was checked by adding a $0.5 \mathrm{ml}$ aliquot of the dialysate to $0.5 \mathrm{ml}$ of a $1 \%(\mathrm{w} / \mathrm{v})$ solution of silver nitrate (Alfa Aesar, USA) and ensuring the absence of any precipitate. The ADA solution was then frozen and lyophilized.

\section{Determination of the degree of oxidation}

The degree of oxidation was determined by measuring the unconsumed sodium metaperiodate before quenching the reaction with ethylene glycol by UV-Vis absorption spectroscopy using a soluble starch indicator. ${ }^{33}$ The indicator solution was prepared by mixing equal volumes of $20 \%(\mathrm{w} / \mathrm{v}) \mathrm{KI}$ and $1 \%(\mathrm{w} / \mathrm{v})$ soluble starch solutions, using phosphate buffer $(\mathrm{pH} 7)$ as solvent. $1 \mathrm{ml}$ aliquot of the reaction mixture was diluted to 250 $\mathrm{ml}$ with DI water before adding the quencher into the reaction. Then $3 \mathrm{ml}$ of the diluted solution were mixed with $1.5 \mathrm{ml}$ of indicator solution and the volume was completed to $5 \mathrm{ml}$ with DI water. The absorbance of the triiodine-starch complex was instantly measured with a UV-Vis spectrophotometer (Specord 40, Analytik Jena, Germany) at $486 \mathrm{~nm}$. The periodate concentration in the sample was obtained using the molar absorption coefficient previously calculated from the absorbance of the complex versus $\mathrm{IO}_{4}^{-}$concentration $\left(0.8-3.5 \times 10^{-5} \mathrm{M}\right)$. The difference between the initial and final amount of $\mathrm{IO}_{4}{ }^{-}$, reacted with sodium alginate and oxidized the corresponding hydroxyl groups, led to transformation into aldehyde groups.

\section{Preparation of hydrogels}

To prepare $5 \%(\mathrm{w} / \mathrm{v})$ ADA and $5 \%(\mathrm{w} / \mathrm{v})$ gelatin solutions, ADA and gelatin were dissolved in phosphate buffered saline (PBS) and ultrapure water, respectively. Gelatin solution was added slowly to the solution of ADA under continuous stirring to facilitate crosslinking between ADA and gelatin. The weight ratios of ADA to gelatin in the final hydrogels were 70/30, 60/40, $50 / 50,40 / 60$ and 30/70 and their compositions are shown in Table 1.

Table 1 Labels used for different samples as a function of their composition

\begin{tabular}{llllll}
\hline \multicolumn{2}{l}{ Weight ratios (\%) } & & \multicolumn{2}{l}{ Final concentration (w/v\%) } & \\
\cline { 1 - 1 } ADA & Gelatin & & ADA & Gelatin & $\begin{array}{l}\text { Labels for } \\
\text { composition }\end{array}$ \\
\hline 70 & 30 & 3.5 & 1.5 & ADA70-GEL30 \\
60 & 40 & 3 & 2 & ADA60-GEL40 \\
50 & 50 & 2.5 & 2.5 & ADA50-GEL50 \\
40 & 60 & 2 & 3 & ADA40-GEL60 \\
30 & 70 & 1.5 & 3.5 & ADA30-GEL70
\end{tabular}




\section{Determination of the degree of crosslinking}

The degree of crosslinking of synthesized ADA-GEL of different compositions was determined by ninhydrin assay. ${ }^{34,35}$ ADA-GEL hydrogels $(1 \pm 0.1 \mathrm{~g})$ were heated with $2 \%(\mathrm{w} / \mathrm{v})$ ninhydrin solution at $100{ }^{\circ} \mathrm{C}$ for $20 \mathrm{~min}$. The optical absorbance of the resulting solution was recorded at $570 \mathrm{~nm}$ using a UV-Vis spectrophotometer (Specord 40, Analytik Jena, Germany). The number of free amino groups in the crosslinked hydrogels which reacted with ninhydrin is proportional to the optical absorbance of the solution. The concentration of free amino groups in the crosslinked hydrogels was determined from a standard curve of glycine concentration vs. absorbance. Alginate-gelatin blend samples without crosslinking were used as control materials. The degree of crosslinking was calculated by the following equation:

$$
\text { Degree of crosslinking }(\%)=\frac{\left(\mathrm{NH}_{2}\right)_{\mathrm{nc}}-\left(\mathrm{NH}_{2}\right)_{\mathrm{c}}}{\left(\mathrm{NH}_{2}\right)_{\mathrm{nc}}} \times 100
$$

where, $\left(\mathrm{NH}_{2}\right)_{\mathrm{nc}}$ and $\left(\mathrm{NH}_{2}\right)_{\mathrm{c}}$ are the mole fractions of free amino groups in non-crosslinked and crosslinked samples, respectively.

\section{Gelation time of the hydrogel}

The test tube inverting method was applied to determine the gelation time according to the procedure reported by Nguyen et $a l .{ }^{36}$ Gelatin solution was slowly added to ADA solution in a narrow beaker (length $7 \mathrm{~cm}$ and inner diameter $3.4 \mathrm{~cm}$ ) under stirring. Chemical coupling between ADA and gelatin occurred during crosslinking. The hydrogel was observed by inverting the beaker and the gelation time was recorded when the hydrogel stopped flowing. The gelation time was recorded at room temperature.

\section{Rheological analysis}

A Discovery Hybrid Rheometer Series 3 (DHR-3) (TA Instruments, USA) was used for the rheological analysis of the ADAGEL hydrogel. A cone plate geometry with a cone diameter of $40 \mathrm{~mm}$ and a cone angle of $2^{\circ}$ was used and the measurement gap was fixed at $65 \mu \mathrm{m}$. A Peltier plate with a diameter of $40 \mathrm{~mm}$ combined with a thermostat was used to maintain the temperature at $25{ }^{\circ} \mathrm{C}$. A solvent trap was used during the measurement to prevent drying of the sample. The oscillation time sweep experiment was performed to record the storage modulus $\left(G^{\prime}\right)$ and loss modulus $\left(G^{\prime \prime}\right)$ of ADA50-GEL50 to achieve the crosslinking point. The oscillation frequency was set at $10 \mathrm{rad} \mathrm{s}^{-1}$ and applied shear stress at $2 \mathrm{~Pa}$.

\section{FTIR analysis}

A Fourier transform infrared (FTIR) spectrometer (Nicolet 6700, Thermo Scientific, USA) was used to evaluate the crosslinking between alginate and gelatin. Dried films were used to record attenuated total reflectance Fourier transform infrared (ATRFTIR) spectra. Films of alginate, gelatin and ADA-GEL materials were made by casting the corresponding hydrogels into a polystyrene Petri dish and allowed to dry for 3 days at room temperature.

\section{NMR analysis}

Solid-state NMR (nuclear magnetic resonance) experiments of alginate and ADA were carried out using a Bruker AVANCE 400 spectrometer with a frequency of $100.613 \mathrm{MHz}$ (magnetic field strength $9.4 \mathrm{~T}$ ) for ${ }^{13} \mathrm{C}$. The experiments were carried out using a $4 \mathrm{~mm}$ MAS (magic angle spinning) probe at a spinning speed of $10 \mathrm{kHz}$ at room temperature $(298 \mathrm{~K}) .{ }^{13} \mathrm{C}$ CPMAS NMR (crosspolarization under magic angle spinning nuclear magnetic resonance) spectra were recorded using a $90^{\circ}$ pulse of $7.2 \mu$ s and a contact time of $2 \mathrm{~ms}$. The recycle delays for alginate and ADA were $2 \mathrm{~s}$ and $4 \mathrm{~s}$, respectively. All the spectra were referenced to tetramethylsilane (TMS) using tyrosine hydrochloride (carboxylic carbon 172.48 ppm from TMS) as the secondary reference for ${ }^{13} \mathrm{C}$.

\section{X-ray diffraction}

The diffraction patterns of alginate, gelatin, ADA and ADA-GEL (different compositions) dried films were analyzed by X-ray scattering using a Rigaku Ultima+ diffractometer using $\mathrm{Cu} \mathrm{K} \alpha$ radiation. The diffraction intensity was recorded within the range of $5-50^{\circ}$ at a scanning rate of $5^{\circ} \mathrm{min}^{-1}$.

\section{Thermogravimetric analysis}

Thermogravimetric analysis was carried out with a SETARAM SETSYS TG-DTA 16/18 instrument. Samples were placed in alumina crucibles. An empty alumina crucible was used as a reference. Alginate, ADA, gelatin and ADA-GEL (different compositions) films were heated from ambient temperature to $1000{ }^{\circ} \mathrm{C}$ in a $50 \mathrm{ml} \mathrm{min}{ }^{-1}$ flow of $\mathrm{N}_{2}$ at a heating rate of $10^{\circ} \mathrm{C}$ $\min ^{-1}$. Continuous recordings of sample temperature, sample weight and heat flow were taken. Thermogravimetric analysis experiments were also performed for microcapsules of alginate, ADA and ADA-GEL (different compositions). The microcapsules of each hydrogel were heated in alumina crucibles from ambient temperature to $275^{\circ} \mathrm{C}$ in a $50 \mathrm{ml} \mathrm{min}{ }^{-1}$ flow of $\mathrm{N}_{2}$ at a heating rate of $10^{\circ} \mathrm{C} \mathrm{min}^{-1}$.

\section{Fabrication of microcapsules}

Microcapsules were fabricated by applying a pneumatic extrusion technique. In brief, hydrogels were transferred into an extrusion cartridge (Nordson EFD, USA) and connected to a high precision fluid dispenser (Ultimus V, Nordson EFD, USA). Microcapsules were generated by applying different air pressures ( 0.55 bar to 2.5 bars) and collected in a beaker containing calcium chloride solution $(0.1 \mathrm{M})$ and kept for 10 minutes to allow ionic gelation. Then the fabricated microcapsules were sieved and washed three times with ultrapure water to remove the calcium chloride solution from the surface of the microcapsules.

\section{Morphology of microcapsules}

The microstructure of the microcapsules was investigated using a scanning electron microscope (SEM) (LEO 435 VP, LEO Electron Microscopy Ltd, Cambridge, UK) at $15 \mathrm{kV}$. The samples were prepared for SEM analysis by drying with a critical point 
dryer (Leica EM CPD300, Germany) and sputter-coated with gold before examination.

\section{Swelling behavior of microcapsules}

Swelling studies of microcapsules were performed in tris buffered saline solution (TBSS), at pH 7.4 at room temperature. The weight of air dried microcapsules $\left(W_{\mathrm{d}}\right)$ was recorded prior to immersion in TBSS. At different time points, the microcapsules were weighed $\left(W_{\mathrm{s}}\right)$ after removing the excess of TBSS by using sieves and blotting paper. The swelling ratio was calculated by the following equation:

$$
\text { Swelling ratio }(\%)=\frac{\left(W_{\mathrm{s}}-W_{\mathrm{d}}\right)}{W_{\mathrm{d}}} \times 100
$$

\section{Degradation study of microcapsules}

The degradation behavior of fabricated microcapsules of different hydrogels was analyzed by recording the diameter of about 30 microcapsules of each hydrogel at different time points during incubation in TBSS at $37{ }^{\circ} \mathrm{C}$. The diameter of microcapsules was measured by light microscopy (Zeiss, Primovert, Germany).

\section{Gelatin release}

Weighed amounts (50 $\mathrm{mg}$ and $200 \mathrm{mg}$ ) of fabricated ADA-GEL microcapsules were immersed in $5 \mathrm{ml}$ of Hank's balanced salt solution (HBSS) and in $20 \mathrm{ml}$ of TBSS, respectively at $37{ }^{\circ} \mathrm{C}$ and $\mathrm{pH}$ 7.4. At selected time points, the solution was removed and collected for gelatin release analysis, and fresh HBSS $(5 \mathrm{ml})$ and TBSS $(20 \mathrm{ml})$ were added to the $50 \mathrm{mg}$ and $200 \mathrm{mg}$ microcapsules, respectively. The gelatin concentration in the released buffer was determined by colorimetric protein assay by the Lowry method, ${ }^{37}$ with bovine serum albumin (BSA) as a standard. The absorbance of each solution at $750 \mathrm{~nm}$ was measured using a UV-Vis spectrophotometer (Specord 40, Analytik Jena, Germany). The release (\%) of gelatin from the microcapsules was calculated as follows:

$$
\text { Gelatin release }(\%)=\frac{[\text { Gelatin }]_{\text {supernatant }}}{[\text { Gelatin }]_{\text {total }}} \times 100
$$

where, [Gelatin $]_{\text {total }}$ is the initial concentration of gelatin (in microcapsules) and [Gelatin $]_{\text {supernatant }}$ is the final gelatin concentration in the buffer solution at different time points.

\section{Results and discussion}

\section{Oxidation of sodium alginate}

Partial oxidation of alginate by using periodate as the oxidizing agent specifically cleaves the vicinal glycols in polysaccharide to form their dialdehyde derivatives. One molecule of periodate is consumed by each $\alpha$-glycol group and the rate of the reaction is dependent on the stereochemistry of the $\alpha$-glycol group under given conditions. ${ }^{18}$ Sodium alginate was oxidized by sodium metaperiodate in an ethanol-water (1:1) mixture at room temperature for 6 hours under dark conditions. Alginate forms a very viscous solution in aqueous medium even at very low concentration which is very difficult to handle and yields a small quantity of oxidized product. On the other hand a small quantity of solvent is needed to carry out the reaction in the ethanol-water mixture even for getting a large quantity of oxidized product, which makes the reaction handier. It has been reported that the oxidation reaction that occurs in ethanol-water medium gives $50-60 \%$ yield of the oxidized product, as compared to the yield (25-35\%) in aqueous medium. ${ }^{38}$ In the oxidation reaction, hydroxyl groups on carbons 2 and 3 of repetitive units were oxidized by sodium metaperiodate (Scheme 1) leading to the formation of two aldehyde groups in each oxidized monomeric unit by cleaving the carbon-carbon bond. ADA of different oxidation degrees can be obtained by changing the ratio of sodium metaperiodate and alginate. It has been revealed that sodium metaperiodate is not completely consumed during oxidation due to the hemiacetal formation between oxidized and unoxidized alginate residue. ${ }^{39}$ FTIR spectra of sodium alginate, gelatin, ADA and ADA50-GEL50 are shown in Fig. 2. The spectrum of sodium alginate demonstrates the characteristic absorption bands of its polysaccharide structure, $1318 \mathrm{~cm}^{-1}$ (C-O stretching), 1126 $\mathrm{cm}^{-1}$ (C-C stretching), $1021 \mathrm{~cm}^{-1}$ (C-O-C stretching) and 947 $\mathrm{cm}^{-1}$ (C-O stretching). ${ }^{\mathbf{2 , 4 0 , 4 1}}$ The absorption bands at around 1590 and $1414 \mathrm{~cm}^{-1}$ are assigned to asymmetric and symmetric stretching peaks of carboxylate salt groups of alginate. ${ }^{42}$ The symmetric vibration of aldehyde at $1735 \mathrm{~cm}^{-1}$ is not detected in the FTIR spectrum. This can be attributed to hemiacetal formation of free aldehyde groups. ${ }^{43}$ However, the formation of aldehyde groups in ADA was confirmed by ${ }^{13} \mathrm{C}$ NMR spectroscopy, as shown in Fig. 1. ${ }^{13} \mathrm{C}$ CPMAS NMR spectroscopy was performed to characterize alginate and ADA. The carbon spectra

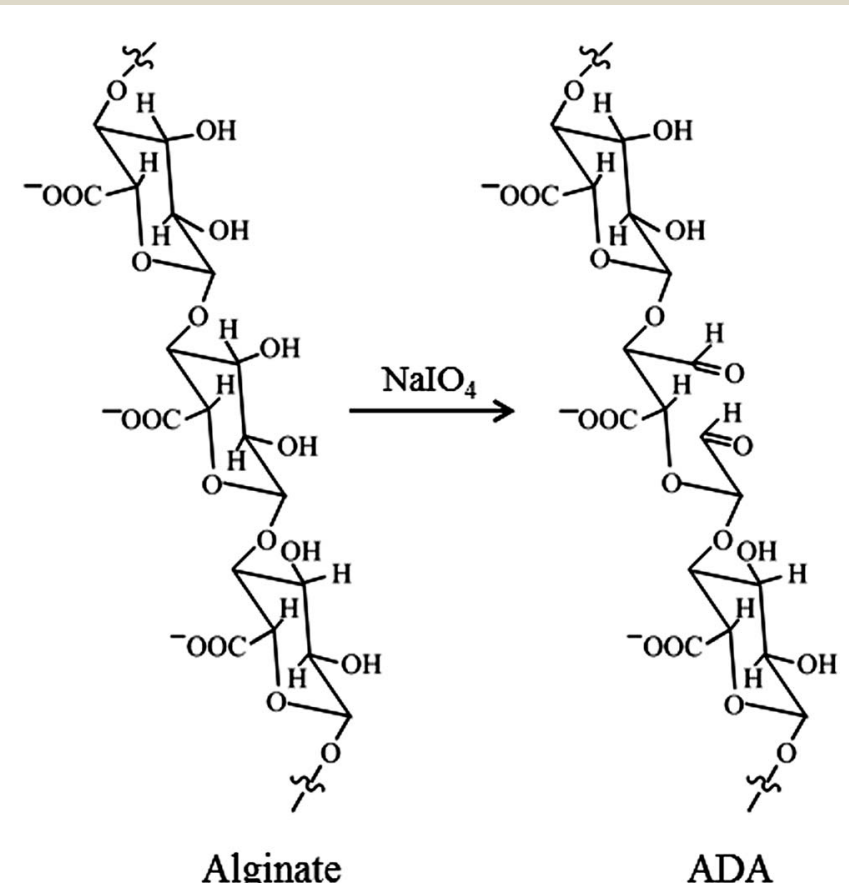

Scheme 1 Scheme for synthesis of ADA from alginate by periodic oxidation. 


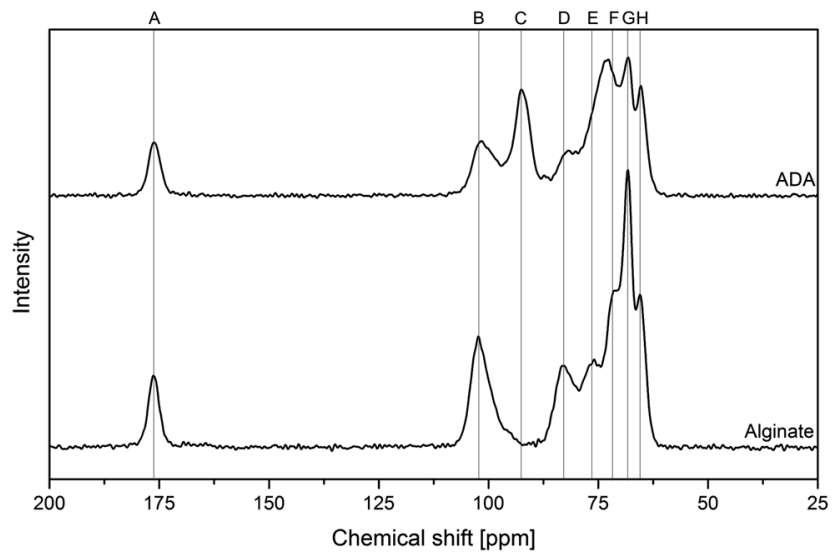

Fig. $1{ }^{13} \mathrm{C}$ CPMAS NMR spectra of alginate and ADA.

of alginate and ADA can generally be divided into three main regions: pyranose carbon (60-90 ppm), anomeric carbon (90$110 \mathrm{ppm})$ and carboxyl carbon (172-180 ppm). ${ }^{44}$ The signals in the spectra were assigned to identify the corresponding carbon of mannuronate (M) and guluronate (G) units of alginate and ADA, according to Salomonsen et al. ${ }^{44,45}$ as presented in Table 2. The signals corresponding to G1, G4, G3 and G5 of ADA became smaller compared to those of alginate. However, the signals corresponding to $\mathrm{M}$ did not change significantly, which indicates that $\mathrm{G}$ units of alginate oxidized preferentially. ${ }^{33}$ Moreover, the new signal at $92.2 \mathrm{ppm}$ appeared in the spectrum of $\mathrm{ADA}$, which can be attributed to hemiacetalic carbons corresponding to aldehyde groups. ${ }^{33}$

\section{Crosslinking of alginate and gelatin}

Crosslinking mainly occurs due to Schiff's base formation through the interaction between the $\varepsilon$-amino groups of lysine or hydroxylysine of gelatin and the available aldehyde groups of ADA (Scheme 2). The degree of crosslinking of ADA-GEL hydrogels was calculated from the moles of the reacted $\varepsilon$-amino groups. The results reported in Table 3 indicate that the ADAGEL hydrogels with lower amounts of gelatin show a higher degree of crosslinking. The degree of crosslinking of ADA50GEL50, ADA40-GEL60 and ADA30-GEL70 decreases significantly. The higher degree of crosslinking of ADA-GEL with lower gelatin content can be explained by the availability of more reactive aldehyde groups of ADA. High ADA content can

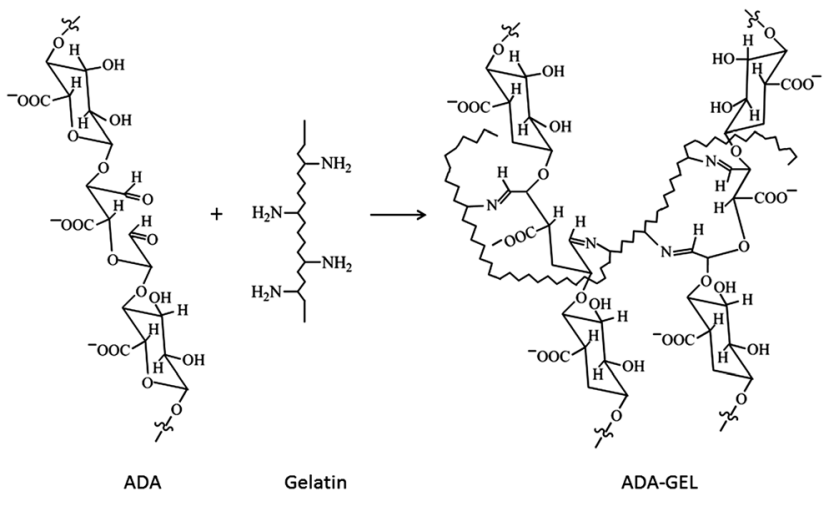

Scheme 2 Schematic presentation of crosslinking of ADA and gelatin and possible coordination of ADA molecules with gelatin chain.

Table 3 Degree of oxidation of ADA obtained by periodate oxidation of sodium alginate and degree of crosslinking of ADA-GEL expressed as percentage of free $\varepsilon$-amino groups consumed after crosslinking as a function of hydrogel composition

\begin{tabular}{llll}
\hline $\begin{array}{l}\text { Periodate } \\
\text { equivalent (wt\%) }\end{array}$ & $\begin{array}{l}\text { Degree of } \\
\text { oxidation } \\
\text { of ADA (\%) }\end{array}$ & Hydrogels & $\begin{array}{l}\text { Degree of } \\
\text { crosslinking (\%) }\end{array}$ \\
\hline & & ADA70-GEL30 & $52.0 \pm 8.2$ \\
32.1 & & ADA60-GEL40 & $51.3 \pm 9.4$ \\
& $33 \pm 0.6$ & ADA50-GEL50 & $34.8 \pm 6.1$ \\
& & ADA40-GEL60 & $40.9 \pm 4.1$ \\
& & ADA30-GEL70 & $36.9 \pm 2.3$
\end{tabular}

promote the extent of crosslinking with the free amino groups of gelatin. On the other hand the ADA50-GEL50, ADA40-GEL60 and ADA30-GEL70 contain 50, 40 and 30\% ADA, respectively which have a comparatively low number of reactive aldehyde groups. Thus a high number of $\varepsilon$-amino groups of gelatin are left uncrosslinked. The FTIR spectrum of gelatin (Fig. 2) shows the characteristic absorption bands of its protein structure, e.g. the bands at 1630 and $1543 \mathrm{~cm}^{-1}$ are assigned to the $\mathrm{N}-\mathrm{H}$ stretching vibration peaks for amide I and amide II, respectively. ${ }^{42,46}$ The spectrum of ADA50-GEL50 (one of the ADA-GEL compositions investigated which is shown representing all compositions as all spectra exhibited the same peaks) presents absorption bands at 1621 and $1557 \mathrm{~cm}^{-1}$ due to $\nu(\mathrm{C}=\mathrm{N})$ suggesting the formation of Schiff's base. ${ }^{47,48}$ The band at

Table 2 Assignments of the resonances in the ${ }^{13} \mathrm{C}$ CPMAS spectra of alginate and ADA (Fig. 1)

\begin{tabular}{|c|c|c|c|c|c|c|c|c|c|c|}
\hline & \multicolumn{10}{|c|}{${ }^{13} \mathrm{C}$ Resonances } \\
\hline & Carboxyl & Anomeric & \multicolumn{7}{|c|}{ Pyranose } & Aldehyde \\
\hline Notation in Fig. 1 & A & B & $\mathrm{D}$ & \multicolumn{2}{|c|}{ E } & \multicolumn{2}{|c|}{$\mathrm{F}$} & G & $\mathrm{H}$ & \multirow[t]{2}{*}{$\mathrm{C}$} \\
\hline Assignment of $\mathrm{M} \& \mathrm{G}^{a}$ & M6 & G1 & G4 & M4 & M5 & M3 & M2 & G3 & G2 & \\
\hline Chemical shift ${ }^{b}$ of alginate (ppm) & 176.2 & 102.4 & 82.8 & & & & & 68.2 & 65.4 & \\
\hline Chemical shift $^{b}$ of ADA (ppm) & 176.1 & 102.2 & 80.9 & \multicolumn{4}{|c|}{72.5} & 68.2 & 65.2 & 92.2 \\
\hline
\end{tabular}

${ }^{a}$ Assignment of mannuronate (M) or guluronate (G) and the number belongs to the position: G2 and M2 are the C2 carbon atoms of the guluronate and mannuronate units, respectively. ${ }^{b}$ The carboxylic resonance at $172.48 \mathrm{ppm}$ from TMS was used as the external reference. 


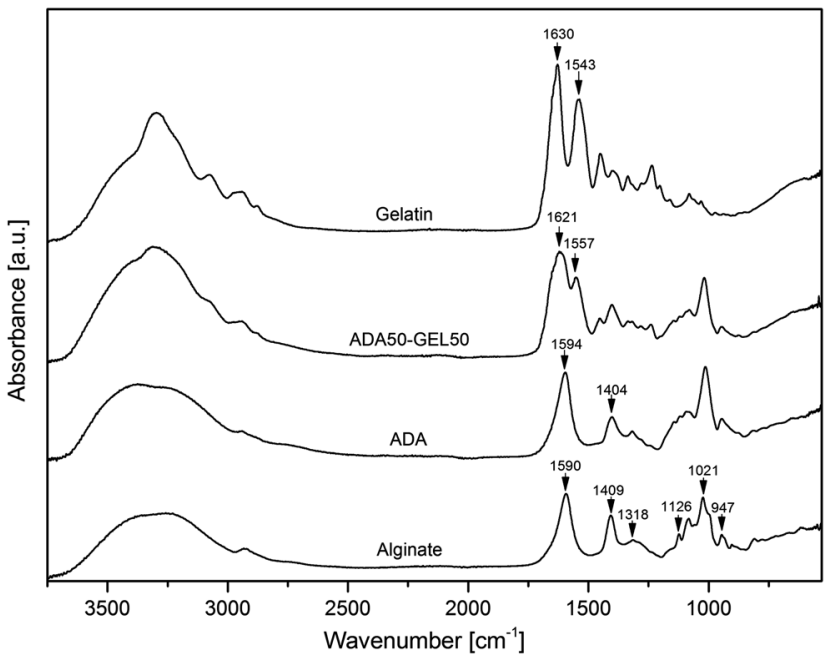

Fig. 2 ATR-FTIR spectra of sodium alginate, ADA, gelatin and ADA50GEL50.

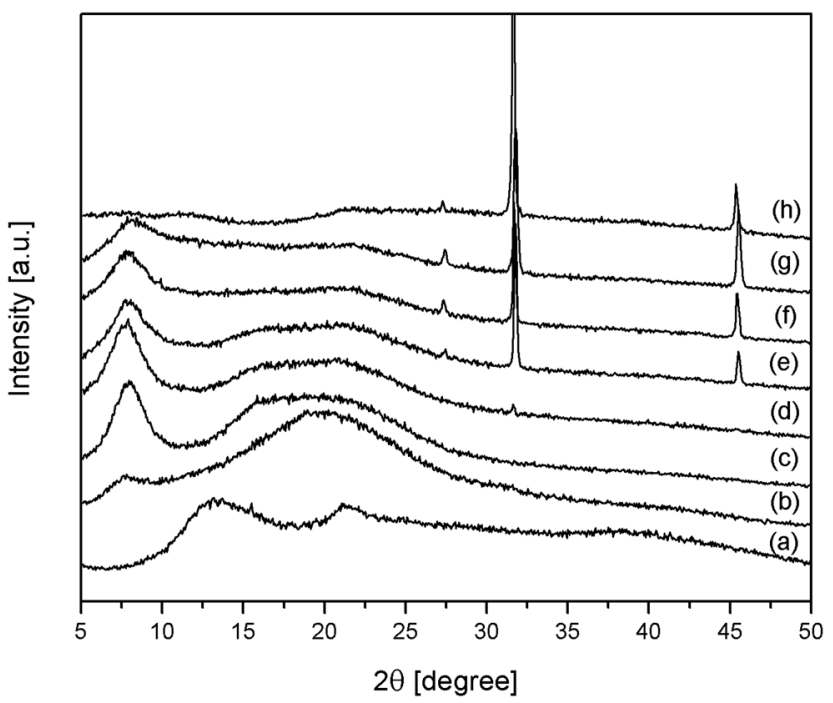

Fig. 3 XRD patterns of (a) alginate, (b) gelatin, (c) ADA30-GEL70, (d) ADA40-GEL60, (e) ADA50-GEL50, (f) ADA60-GEL40, (g) ADA70GEL30 and (h) ADA.
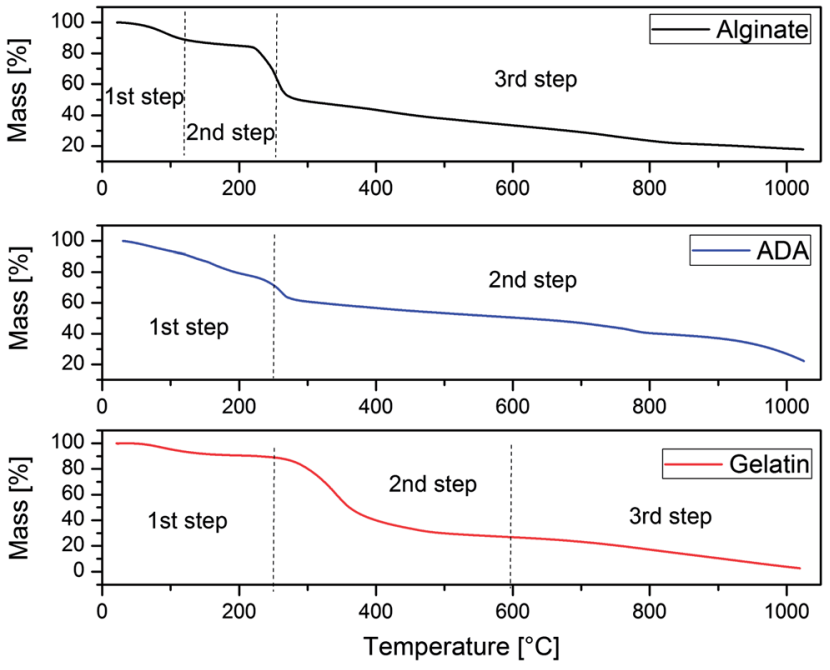

Fig. 5 TG curves of alginate, ADA and gelatin at heating rate of $10^{\circ} \mathrm{Cmin}^{-1}$.

$1621 \mathrm{~cm}^{-1}$ due to Schiff's base is broad probably because of overlapping with the band at $1630 \mathrm{~cm}^{-1}$ of amide I of uncrosslinked gelatin. Moreover, the band at $1543 \mathrm{~cm}^{-1}$ due to amide II, which is characteristic of gelatin, is completely absent in the spectrum of ADA50-GEL50, proving the involvement of this group in the crosslinking reaction.

The X-ray diffraction (XRD) patterns of alginate, gelatin, ADA and ADA-GEL (different compositions), as dried films, are shown in Fig. 3. The diffractogram of alginate consists of two crystalline peaks at $2 \theta=13.4^{\circ}$ and $22.7^{\circ}$ (ref. 49) while the spectrum of gelatin exhibits one wide peak at $2 \theta=20.4^{\circ}$ indicative of its high amorphous content and a small peak at $2 \theta=7.8^{\circ}$, which can be ascribed to the triple-helical crystalline structure of collagen renatured in gelatin. ${ }^{50}$ In addition, the ADA film presents two main peaks at $2 \theta=31.6^{\circ}$ and $45.3^{\circ}$. The diffractograms of the ADA-GEL films are consistent with their composition. Initially, the low-angle peak of gelatin at $2 \theta=7.8^{\circ}$, which is related to the diameter of the triple helix of the collagen molecular structure, seems to be intensified at the diffractograms of the ADA-GEL samples
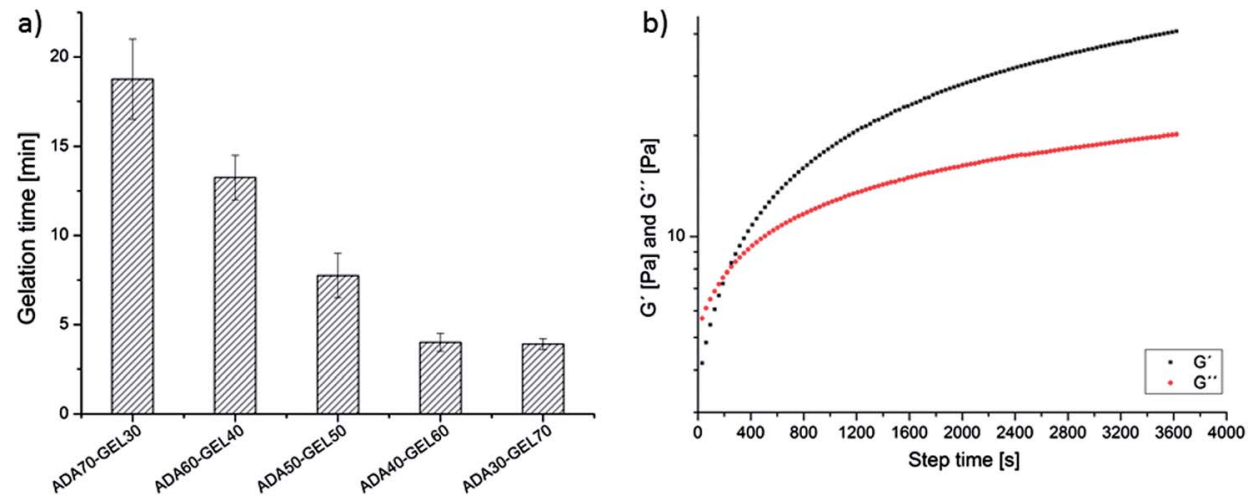

Fig. 4 Gelation time obtained by (a) test tube inverting method of ADA-GEL with different compositions and (b) by analyzing storage moduli (Ǵ) and loss moduli (Ǵ) of ADA50-GEL50 hydrogel as a function of time after mixing of ADA and gelatin under constant shear rate at $25^{\circ} \mathrm{C}$. 

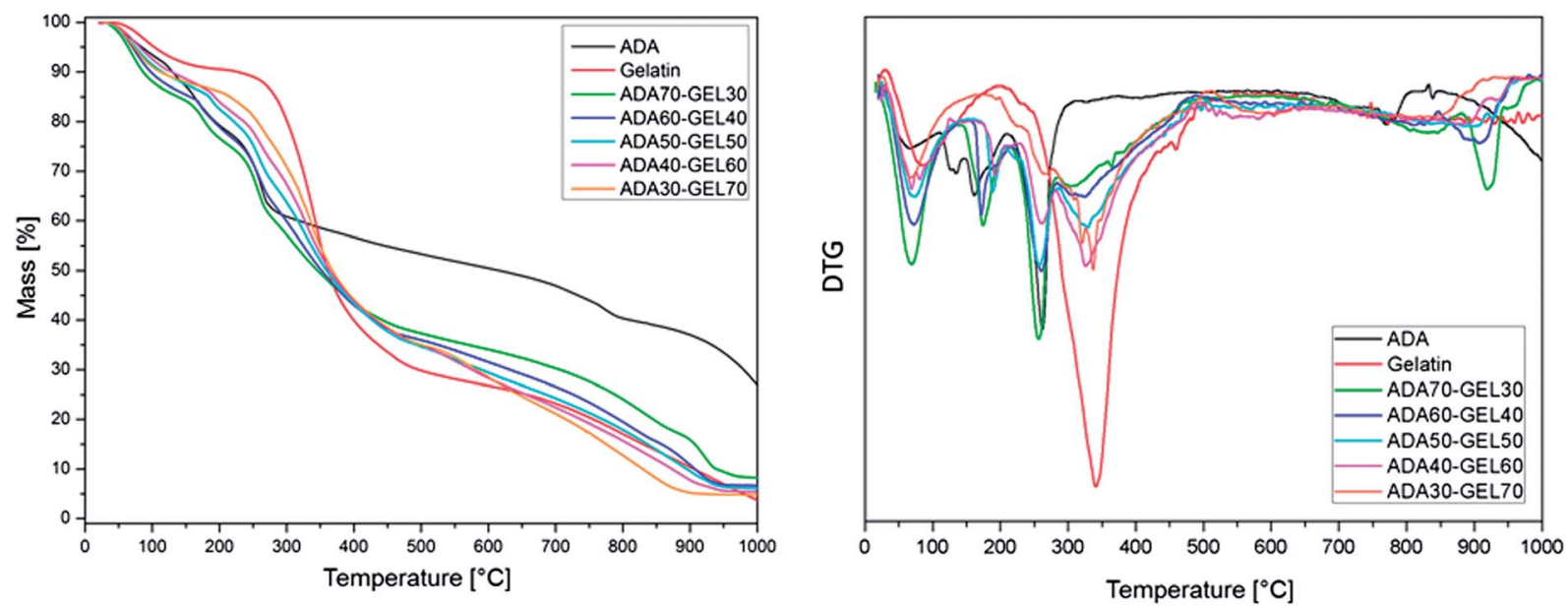

Fig. 6 Mass loss (\%) (left) and derivative thermogravimetric analysis (DTG) (right) curves of dry films fabricated from alginate, ADA and ADA-GEL of different compositions.

and especially for the samples with high gelatin content. This behavior has previously been reported by Boanini et al. ${ }^{3}$ and it is associated with the increase in the content of the triple helix structure of gelatin. In addition, the characteristic peaks of ADA can be observed for the samples containing high ADA content $(>50 \%)$. The intensity of those peaks constantly lowers when the ADA content is reduced, while below the concentration of $50 \%$, the two peaks disappear. Similarly, the broad peak of gelatin at $2 \theta=20.4^{\circ}$ can be detected in the ADA-GEL with high gelatin content $(\geqslant 50 \%)$, while below that concentration the peak almost disappears. The strong interactions between alginate and gelatin have destroyed the close packing for the formation of regular crystallites and this fact can explain the absence of the alginate characteristic peak at $2 \theta=13.4^{\circ}$ in the ADA-GEL samples. Furthermore, the decreased intensity of the peaks in the diffractograms of ADA-GEL indicates a reduction in the degree of crystallinity and this is likely due to the covalent crosslinking between gelatin and ADA.

\section{Gelation time}

The gelation time is defined as the time required for the completion of crosslinking reaction between ADA and gelatin. After mixing the ADA and gelatin solution, an opaque viscous hydrogel was formed after a certain time, which varies for different compositions of ADA and gelatin. Fig. 4a shows the gelation time of ADA-GEL with different compositions of ADA and gelatin. The gelation time of ADA-GEL decreased with increasing gelatin content in ADA-GEL, which ranged from 19 to 4 minutes. However, the gelation time of ADA40-GEL60 and ADA30-GEL70 presents similar values. The gelation time of ADA50-GEL50 is found to be around 8 minutes, presenting a similar value to that obtained with rheological analysis. In the time sweep study of rheological analysis (Fig. 4b), the storage modulus $\left(G^{\prime}\right)$ and loss modulus $\left(G^{\prime \prime}\right)$ were monitored with respect to time in order to determine the rate and extent of crosslinking. The crossover point of $G^{\prime}$ and $G^{\prime \prime}$, where $G^{\prime}$ is equal to $G^{\prime \prime}$, is defined as the state of gel formation..$^{30,51}$ For ADA50GEL50, the crossover point of $G^{\prime}$ and $G^{\prime \prime}$ was found to be about
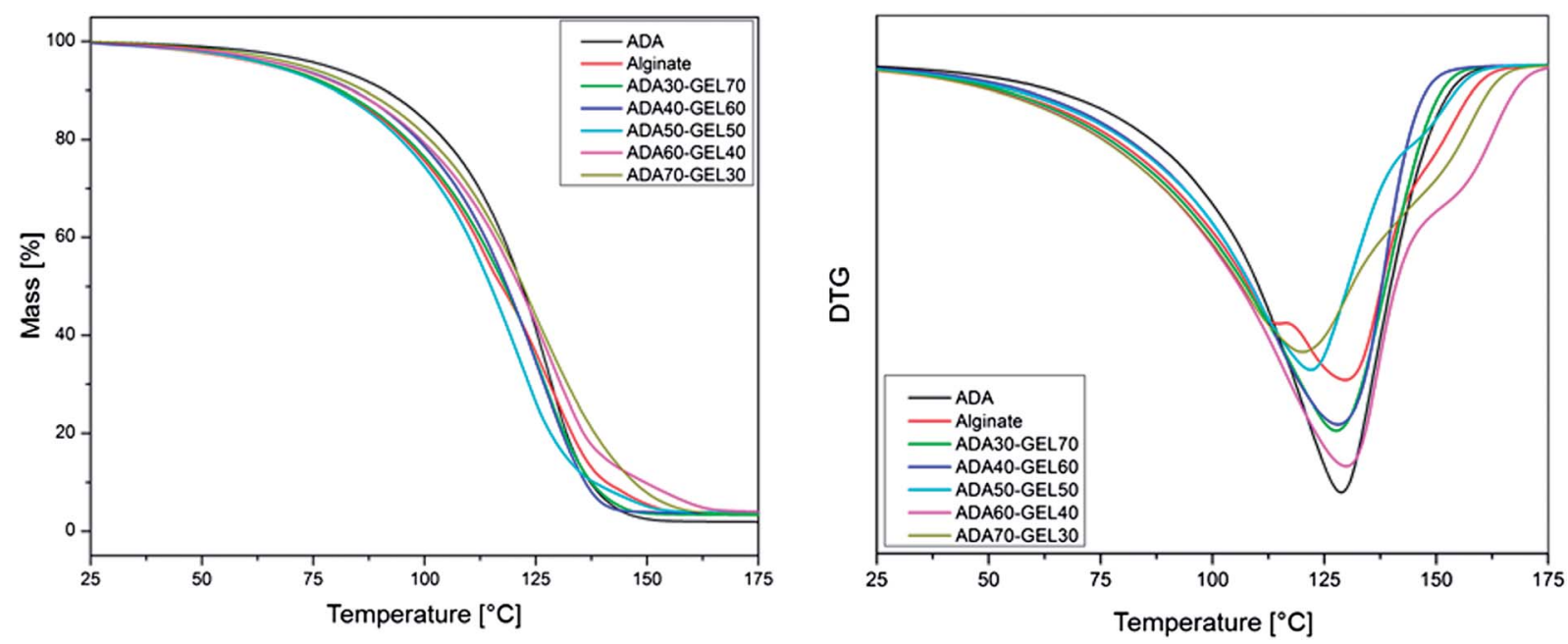

Fig. 7 Mass loss (\%) (left) and DTG (right) curves of microcapsules fabricated from alginate, ADA and ADA-GEL of different compositions. 

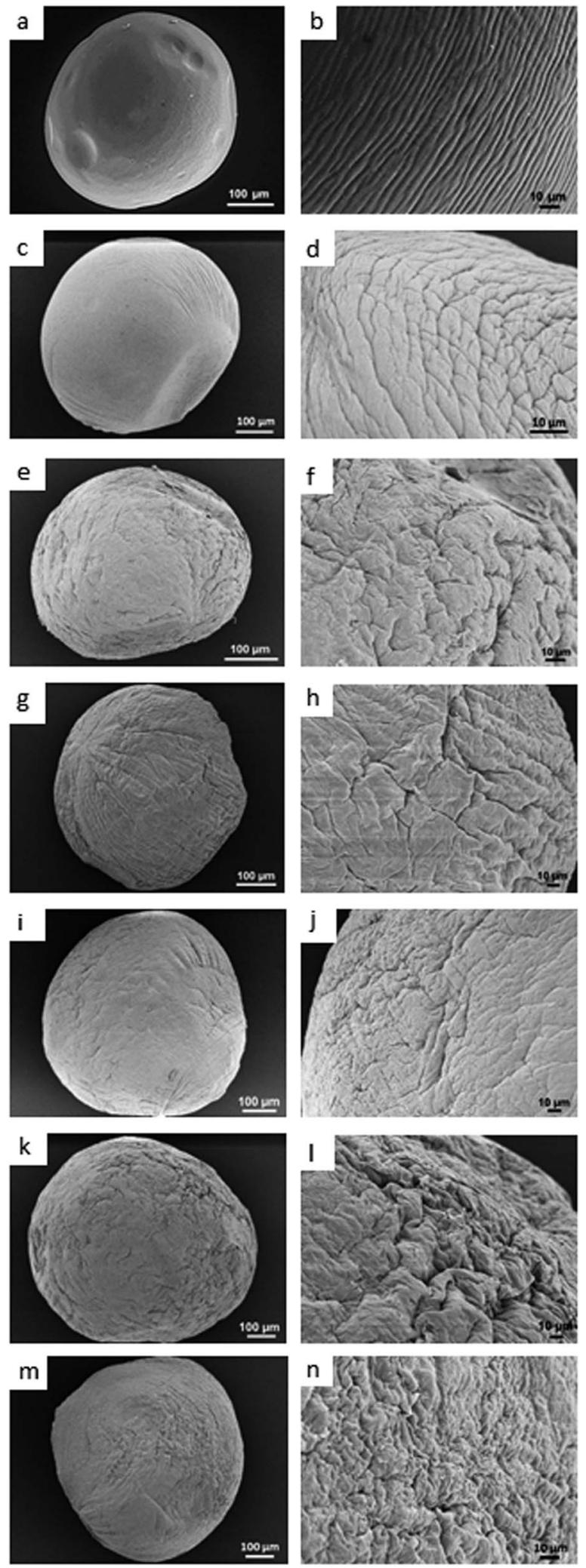

Fig. 8 SEM images of microcapsules fabricated from $2.5 \%(\mathrm{w} / \mathrm{v})$ alginate (a: whole and b: surface), 2.5\% (w/v) ADA (c: whole and d: surface), ADA70-GEL30 (e: whole and f: surface), ADA60-GEL40 (g: whole and h: surface), ADA50-GEL50 (i: whole and j: surface), ADA40-GEL60 (k: whole and l: surface), ADA30-GEL70 ( $\mathrm{m}$ : whole and $\mathrm{n}$ : surface). Scale bar: $100 \mu \mathrm{m}$ (left column); $10 \mu \mathrm{m}$ (right column).
220 seconds (about 3.7 minutes) and the processing time (time required to mix ADA and gelatin and to start the experiment) was about 4.5 minutes. Thus the total time required to achieve the crossover point was about 8.2 minutes, which is almost similar to the value that was observed during the experiment of gelation time measurement of ADA50-GEL50 by the test tube inverting method.

\section{Thermogravimetric analysis}

During the thermogravimetric (TG) analysis of the dried films, the samples were heated up to $1000{ }^{\circ} \mathrm{C}$ with a heating rate of $10{ }^{\circ} \mathrm{C} \min ^{-1}$ under a $\mathrm{N}_{2}$ atmosphere. The mass loss curves of sodium alginate, ADA and gelatin are presented in Fig. 5 .

From the thermograms, it can be seen that all three materials exhibit different thermal behavior. Initially sodium alginate presents three degradation steps. The first one occurs at almost $120{ }^{\circ} \mathrm{C}$ and corresponds to the dehydration of the sample, while the second occurs at an inflection point of $250{ }^{\circ} \mathrm{C}$ and indicates the decomposition of alginate by the dehydration of the saccharide rings, breaking up of $\mathrm{C}-\mathrm{H}$ bonds and breaking of the $\mathrm{C}-\mathrm{O}-\mathrm{C}$ glycoside bonds in the main polysaccharide chain. During the third and final degradation step, $\mathrm{Na}_{2} \mathrm{CO}_{3}$ and other carbonaceous materials were formed. ${ }^{52}$ ADA exhibits different thermal behavior. The first degradation step of ADA is quite fast and corresponds to the elimination of absorbed and bound water from the samples along with low molecular mass compounds. The same applies also for the first degradation step of gelatin, at significantly slower rates. In addition, the second and main degradation stage of ADA is probably associated with the structural degradation of the main chain along with the liberation of volatile compounds. According to Pawlicka et al. ${ }^{53}$ this degradation step corresponds to endothermic hydrolysis and the oxidation process followed by an exothermic reaction, which starts at $345^{\circ} \mathrm{C}$ and corresponds to the final pyrolysis. On the other hand, the second decomposition stage of gelatin is related to the main degradation and represents a complex process including protein chain breakage and peptide bond rupture. ${ }^{54}$ Finally, the third degradation step of gelatin can be

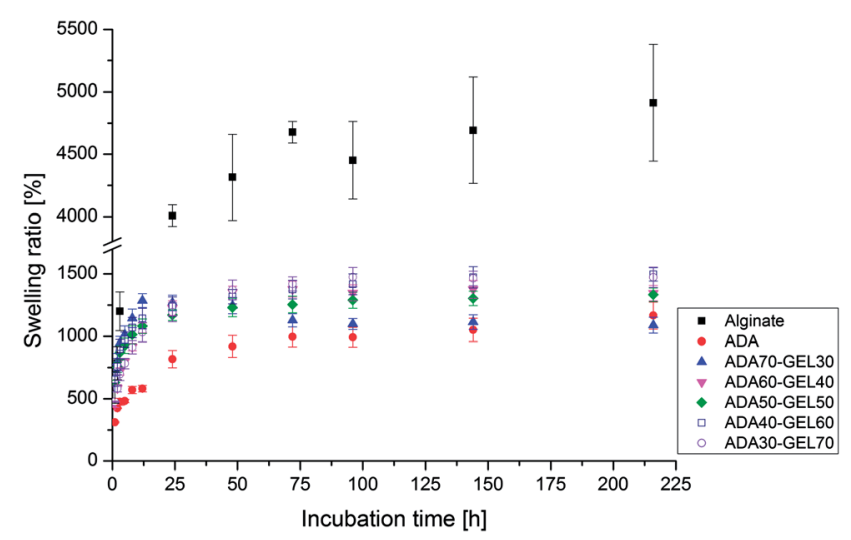

Fig. 9 Swelling ratio (\%) in TBSS as a function of incubation time of the different microcapsules fabricated from alginate; ADA and ADA-GEL hydrogels of different compositions. 
attributed to the decomposition of more thermally stable structures which were produced from crosslinking reactions during heating. The decomposition curves of the ADA-GEL films (Fig. 6) demonstrate that they are not strictly the sum of the combination of the two independent starting materials, ADA and gelatin.

For the ADA-GEL films with high ADA content (ADA70GEL30 and ADA60-GEL40), the thermal stability is lower compared to other compositions for the temperatures up to $370{ }^{\circ} \mathrm{C}$. However, the thermal stability of ADA-GEL films with high content of ADA is enhanced at higher temperatures $\left(>370{ }^{\circ} \mathrm{C}\right)$. On the other hand, the composites which consist of high amounts of gelatin (ADA30-GEL70 and ADA40-GEL60) show exactly the opposite behavior. At lower temperatures $\left(<370{ }^{\circ} \mathrm{C}\right)$, their thermal stability is higher than that of the other ADA-GEL films. Higher amounts of gelatin remained uncrosslinked in ADA30-GEL70 and ADA40-GEL60, and this is the reason why these hydrogels exhibit higher thermal stability at lower temperatures. However, after that point, their thermal stability drops significantly. It is interesting to notice that for the ADA-GEL films with $60 \%$ and $70 \%$ gelatin, the thermal stability was found to be even lower than that of pure gelatin at higher temperatures $\left(>653{ }^{\circ} \mathrm{C}\right)$. This phenomenon is also observed at the primary stages of degradation of the ADA-GEL films with high ADA concentration, which present low thermal stability, even lower than that of their two components, ADA and gelatin. The consistency of the samples is once again confirmed by the fact that the presence of ADA and gelatin has pronounced effects on the patterns of the TG curves of ADAGEL films, depending on the concentration of each component during the whole degradation process. Thus, at the early stages of degradation, the slopes of the TG curves of ADA-GEL films with high ADA content are steeper than those with high gelatin content, while at the end of the degradation process the break of the TG curve which is pronounced at ADA is also visible for the ADA-GEL but at higher temperatures.

Thermogravimetric analysis experiments were also performed for microcapsules of alginate and ADA-GEL of different compositions, the results are presented in Fig. 7. It is obvious from Fig. 7 that the microcapsules degrade very fast and have lost almost all their mass for temperatures up to $175^{\circ} \mathrm{C}$. This is an indication that the microcapsules contain a huge amount of water (almost 94-98\% w/w) which is evaporated at around $100{ }^{\circ} \mathrm{C}$ and a small amount of materials which degrade after a while. This conclusion is further confirmed by the derivative of the mass loss signal, where a shoulder appears at $160{ }^{\circ} \mathrm{C}$.

\section{Morphology of microcapsules}

The surface morphology of microcapsules of alginate, ADA and ADA-GEL of different compositions was evaluated by scanning electron microscopy (SEM) (Fig. 8). Alginate microcapsules exhibit a folded surface structure in regular patterns. The surface becomes less folded and the folding pattern was different for the microcapsules of ADA due to partial oxidation. ADA-GEL microcapsules of all compositions show a totally inhomogeneous surface morphology without any regular patterns of folding.
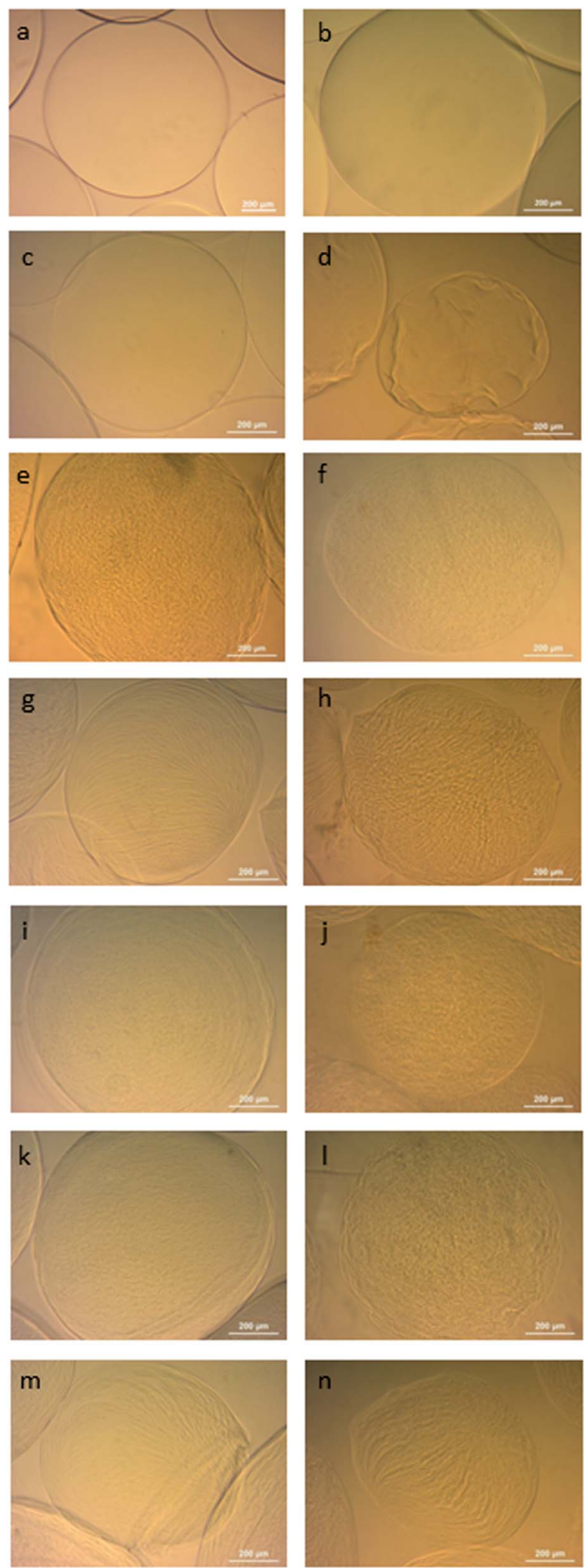

Fig. 10 Light microscopy images of microcapsules fabricated from (a) $2.5 \%(\mathrm{w} / \mathrm{v})$ alginate, (c) 2.5\% (w/v) ADA, (e) ADA70-GEL30, (g) ADA60GEL40, (i) ADA50-GEL50, (k) ADA40-GEL60, (m) ADA30-GEL70 after 0 day of incubation in TBSS and (b) $2.5 \%(\mathrm{w} / \mathrm{v})$ alginate, (d) $2.5 \%(\mathrm{w} / \mathrm{v})$ ADA, (f) ADA70-GEL30, (h) ADA60-GEL40, (j) ADA50-GEL50, (l) ADA40-GEL60, (n) ADA30-GEL70 after 28 days of incubation in TBSS. Scale bar: $200 \mu \mathrm{m}$. 
However, microcapsules of ADA40-GEL60 and ADA30-GEL70 show comparatively higher surface roughness than those of other compositions of ADA-GEL. The inhomogeneous morphology of ADA-GEL microcapsules was attributed to the crosslinking reaction between ADA and gelatin which destroyed the regular structural pattern of alginate as it was also shown by the XRD results.

\section{Swelling behavior of microcapsules}

The swelling ratio of microcapsules of alginate and ADA-GEL of different compositions was analyzed in TBSS which represents the ability of the microcapsules to keep and diffuse buffer solution and it is related to the ability of the capsules to absorb body fluid and transfer cell nutrients and metabolites. This is one of the most important properties of hydrogel based biomaterials. ${ }^{36,55}$ As shown in Fig. 9, the swelling ratio of ADA microcapsules decreased significantly compared to alginate microcapsules. This behavior could possibly be because ADA is a partially oxidized product of alginate, which forms by cleaving vicinal glycols of alginate and decreasing its molecular weight leading to lower water keeping ability. The swelling ratios of ADA and ADA-GEL microcapsules increased rapidly up to 24 hours and then a plateau is reached. However, for alginate capsules, the swelling ratio increased rapidly up to 72 hours. For longer periods of incubation, ADA-GEL microcapsules with high concentration of ADA showed a comparatively lower swelling ratio than that of the microcapsules of high gelatin content. This result is related to the swelling behavior of ADA and it is in agreement with the results reported by Boanini et al. ${ }^{3}$ This behavior could possibly be due to the relatively low stability of ADA in TBSS.

\section{Degradation behavior of microcapsules}

Light microscopy images of as-fabricated and 28 days incubated (in TBSS) microcapsules from alginate, ADA and ADA-GEL of different compositions are shown in Fig. 10. The texture observed on the ADA-GEL microcapsules (all compositions) is different from that of microcapsules of alginate and ADA. Microcapsules of alginate and ADA appear smooth and clear. However, the microcapsules of all compositions of ADA-GEL show a crimpy and fuzzy texture which could possibly be due to the crosslinking between ADA and gelatin. The degradation of ADA microcapsules is clearly visible after 28 days of incubation in TBSS.

The degradation properties of microcapsules fabricated from alginate, ADA and different compositions of ADA-GEL were evaluated in terms of diameter changing during incubation in TBSS at $37{ }^{\circ} \mathrm{C}$ (Fig. 11). If the diameter of microcapsules decreased over the incubation time compared to the initial diameter (at day 0), we considered that degradation of the hydrogel microcapsules has occurred. As shown in the figure, ADA microcapsules show higher degradation than alginate microcapsules because of the lower molecular weight of ADA due to cleavage of vicinal glycols during partial oxidation of alginate. ADA and ADA70-GEL30 microcapsules exhibit almost similar degradation patterns over the incubation time in TBSS, due to the fact that having higher amounts of ADA in ADA70GEL30 microcapsules can lead to faster degradation in TBSS. On the other hand, ADA30-GEL70 microcapsules also exhibited a higher degree of degradation. This result can be due to the release of more gelatin which remains uncrosslinked in the high gelatin content ADA-GEL microcapsules. It is therefore confirmed that some ADA-GEL compositions exhibited higher in vitro degradation compared to alginate. Degradation of biomaterials is an important phenomenon for the application in tissue engineering. ${ }^{20}$ For the case of ADA-GEL microcapsules, it is possible to readily control their degradation kinetics by tuning their composition, degree of oxidation of ADA and degree of crosslinking..$^{21,36}$

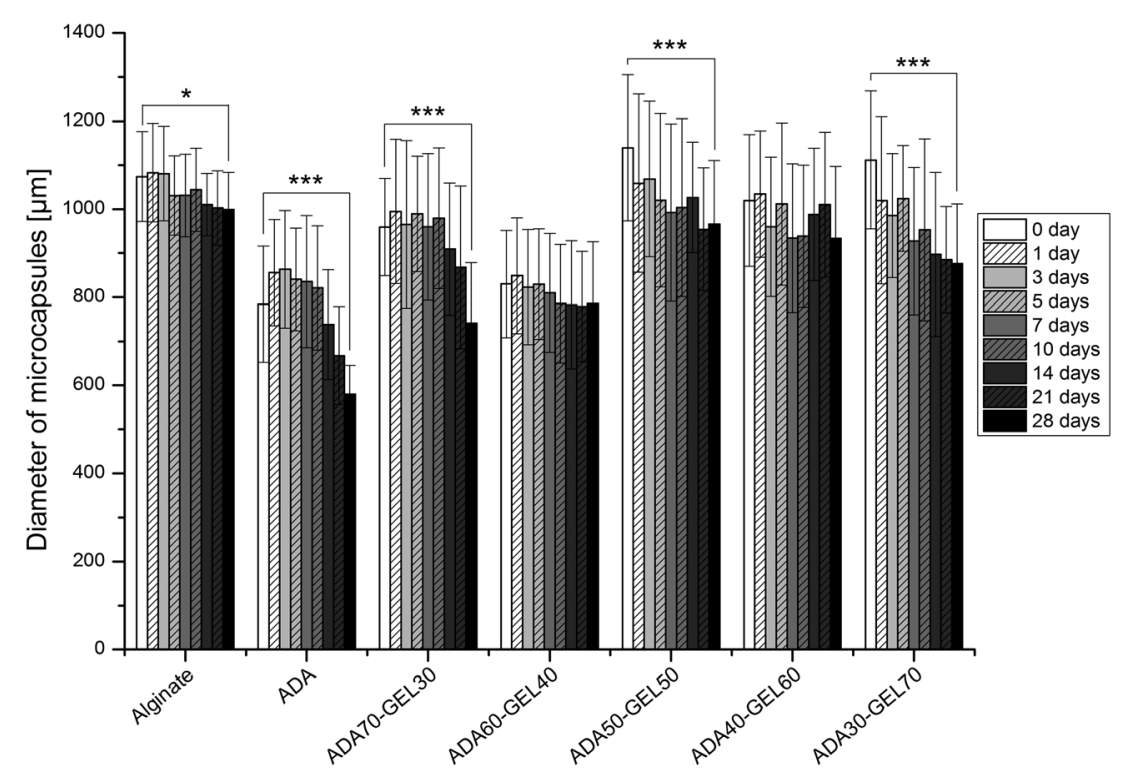

Fig. 11 Variation of the diameter of microcapsules fabricated from alginate, ADA and ADA-GEL of different compositions as a function of incubation time in TBSS. Asterisks denote significant difference, ${ }^{*} p<0.05, * * p<0.01$ and $* * * p<0.001$ (Bonferroni's post-hoc test was used). 
a)

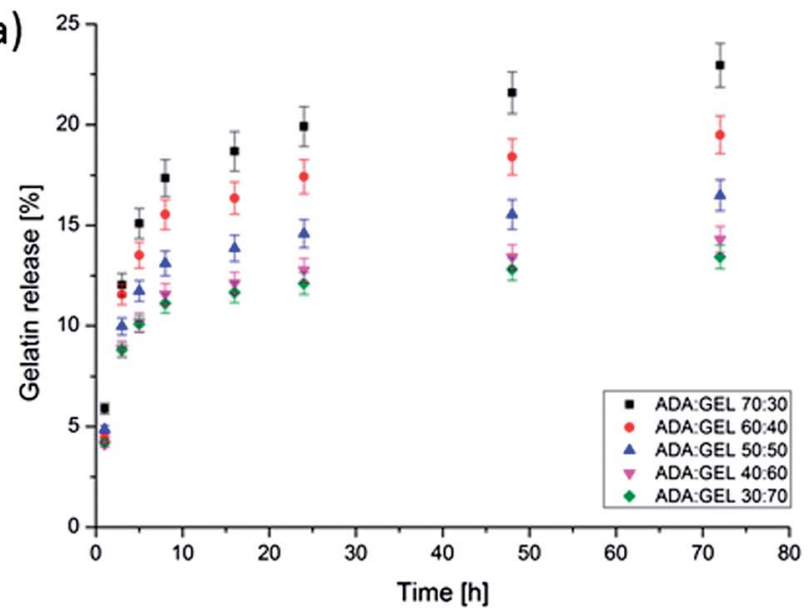

b)

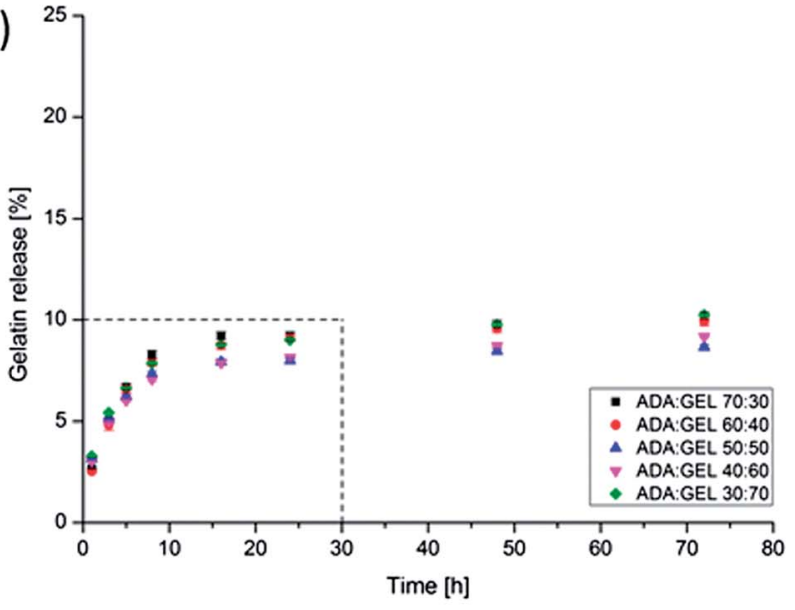

$b^{\prime}$

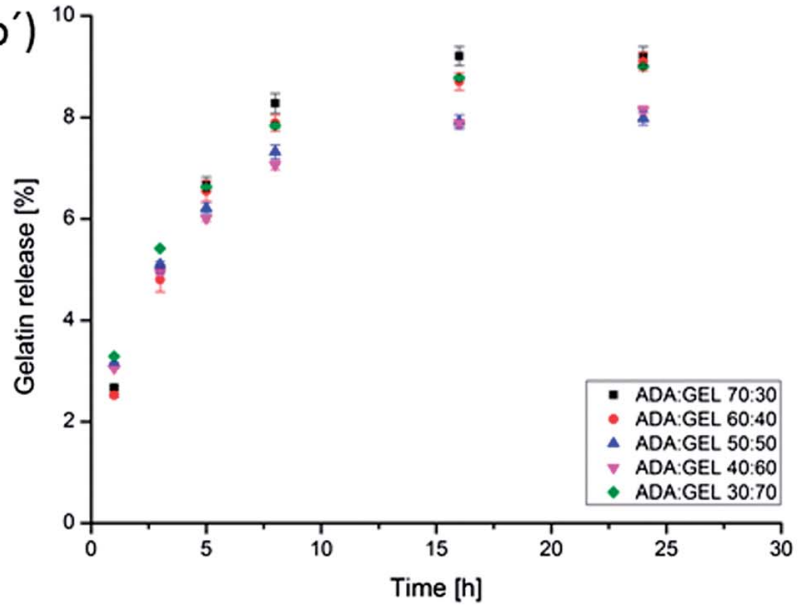

Fig. 12 Cumulative gelatin release (wt\%) as a function of incubation time in (a) TBSS and (b) HBSS of the microcapsules fabricated from ADA-GEL hydrogels of different compositions. (b́) enlarged view of selected area of graph (b).

\section{Gelatin release}

The gelatin release of ADA-GEL microcapsules of different compositions in HBSS and TBSS at pH 7.4 at $37{ }^{\circ} \mathrm{C}$ (Fig. 12) was monitored to investigate the effect of different buffer solutions on gelatin release behavior of ADA-GEL microcapsules. The release of gelatin started immediately after immersion in both buffer solutions. Initially the release rate was high in both buffer solutions, but after a certain time, the release rate decreased. The high release rate of gelatin from microcapsules in HBSS and TBSS was found to be maintained up to $16 \mathrm{~h}$ and 8 $\mathrm{h}$, respectively. However, the release amount of gelatin was found to be higher in TBSS than in HBSS. After 24 hours gelatin release values reached about 9, 9, 8, 8 and $9 \mathrm{wt} \%$ in HBSS and about 20, 17, 15, 13 and $12 \mathrm{wt} \%$ in TBSS for ADA70-GEL30, ADA60-GEL40, ADA50-GEL50, ADA40-GEL60 and ADA30GEL70, respectively. The reduction of gelatin release on increasing gelatin concentration in ADA-GEL microcapsules might be explained by the increasing extent of physical as well as chemical crosslinking in this material. ${ }^{3}$ In both buffer solutions, namely HBSS and TBSS, the percentage of released gelatin was found to be very low which means that a significant quantity of gelatin remained in the microcapsules that could enhance cell-material interactions due to the presence of the cell adhesive peptide, RGD (Arg-Gly-Asp) sequence, in gelatin. ${ }^{56,57}$ This result thus indicates that the present ADA-GEL microcapsules could be very promising for tissue engineering applications. Moreover, a comparatively higher amount of gelatin was released from all of the compositions of ADA-GEL microcapsules in TBSS than in HBSS. This phenomenon might be ascribed to the presence of calcium salt in HBSS but not in TBSS. Thus the calcium ions in HBSS interact with the hydrogel increasing the stability of the alginate based microcapsules. On the other hand, the microcapsules are less stable in sodium salt base buffer solution, e.g. TBSS. Moreover, it was observed that the gelatin release percentage was increased in TBSS with the increase of ADA content in ADA-GEL microcapsules. This behavior could be assigned to the instability of ADA in TBSS and the lower extent of physical and chemical crosslinking due to low amounts of gelatin.

\section{Conclusions}

ADA-GEL were synthesized by covalent bonding between ADA and gelatin. For the first time, microcapsules were successfully fabricated from different compositions of ADA-GEL by tuning the oxidation degree of ADA, crosslinking degree and gelation time of ADA-GEL. Microcapsules from all compositions of ADAGEL showed higher degradability compared to alginate microcapsules and this fact is of importance for application in tissue engineering. SEM images showed a rougher and irregular surface morphology of ADA-GEL microcapsules compared to alginate microcapsules which is related to the higher degradation behavior of ADA-GEL in comparison to alginate. In most of the previous studies, ADA-GEL has been synthesized in the presence of borax that increases the $\mathrm{pH}$ of the reaction medium and reduces the gelation time of the hydrogel. However it is not possible to fabricate microcapsules within this short gelation time and moreover high $\mathrm{pH}$ could inhibit cell viability. In the present study, we synthesized ADA-GEL in PBS which maintained the $\mathrm{pH}$ at around 7.4 that increased the gelation time of hydrogel that facilitated the fabrication of microcapsules. Moreover the $\mathrm{pH}$ around 7.4 is helpful for better cell attachment, migration and proliferation. The study of the fabrication 
of more round and smaller microcapsules of ADA-GEL by controlling its degree of crosslinking and gelation properties is in progress. The biocompatibility of the hydrogel in 3-D needs to be evaluated by encapsulating different types of cells, which constitutes the focus of the current research.

\section{Acknowledgements}

This work was supported by the Emerging Fields Initiative (EFI) of the University of Erlangen-Nuremberg (project TOPbiomat) and the German Academic Exchange Service (DAAD).

\section{References}

1 K. H. Bouhadir, D. S. Hausman and D. J. Mooney, Polymer, 1999, 40, 3575-3584.

2 E. F. S. Vieira, A. R. Cestari, C. Airoldi and W. Loh, Biomacromolecules, 2008, 9, 1195-1199.

3 E. Boanini, K. Rubini, S. Panzavolta and A. Bigi, Acta Biomater., 2010, 6, 383-388.

4 J. L. Wilson and T. C. McDevitt, Biotechnol. Bioeng., 2013, 110, 667-682.

5 H.-B. Li, H. Jiang, C.-Y. Wang, C.-M. Duan, Y. Ye, X.-P. Su, Q.-X. Kong, J.-F. Wu and X.-M. Guo, Biomed. Mater., 2006, 1, 42-47.

6 S. A. Abbah, W. W. Lu, D. Chan, K. M. C. Cheung, W. G. Liu, F. Zhao, Z. Y. Li, J. C. Y. Leong and K. D. K. Luk, J. Mater. Sci. Mater. Med., 2008, 19, 2113-2119.

7 S. Sakai, S. Ito and K. Kawakami, Acta Biomater., 2010, 6, 3132-3137.

8 N. E. Fedorovich, E. Kuipers, D. Gawlitta, W. J. A. Dhert and J. Alblas, Tissue Eng., Part A, 2011, 17, 2473-2486.

9 T. Billiet, M. Vandenhaute, J. Schelfhout, S. Van Vlierberghe and P. Dubruel, Biomaterials, 2012, 33, 6020-6041.

10 J.-H. Shim, J.-S. Lee, J. Y. Kim and D.-W. Cho, J. Micromech. Microeng., 2012, 22, 085014.

11 F. P. W. Melchels, M. A. N. Domingos, T. J. Klein, J. Malda, P. J. Bartolo and D. W. Hutmacher, Prog. Polym. Sci., 2012, 37, 1079-1104.

12 S. Wüst, R. Müller and S. Hofmann, J. Funct. Biomater., 2011, 2, 119-154.

13 I. W. Sutherland, Biomaterials: Novel Materials from Biological Sources, ed. D. Byrom, Stockton Press, New York, 1991, pp. 309-331.

14 T. Boontheekul, H.-J. Kong and D. J. Mooney, Biomaterials, 2005, 26, 2455-2465.

15 H. Kong, M. K. Smith and D. J. Mooney, Biomaterials, 2003, 24, 4023-4029.

$16 \mathrm{~J}$. A. Rowley, G. Madlambayan and D. J. Mooney, Biomaterials, 1999, 20, 45-53.

17 A. Leal-Egaña, U.-D. Braumann, A. Díaz-Cuenca, M. Nowicki and A. Bader, J. Nanobiotechnol., 2011, 9, 24.

18 B. Balakrishnan and A. Jayakrishnan, Biomaterials, 2005, 26, 3941-3951.

19 H. Liao, H. Zhang and W. Chen, J. Mater. Sci. Mater. Med., 2009, 20, 1263-1271.
20 B. Balakrishnan, M. Mohanty, P. R. Umashankar and A. Jayakrishnan, Biomaterials, 2005, 26, 6335-6342.

21 K. H. Bouhadir, K. Y. Lee, E. Alsberg, K. L. Damm, K. W. Anderson and D. J. Mooney, Biotechnol. Prog., 2001, 17, 945-950.

22 E. Boanini and A. Bigi, J. Colloid Interface Sci., 2011, 362, 594599.

23 A. Bigi, S. Panzavolta and K. Rubini, Biomaterials, 2004, 25, 5675-5680.

24 S. B. Ross-Murphy, Polymer, 1992, 33, 2622-2627.

25 I. Pezron, M. Djabourov and J. Leblond, Polymer, 1991, 32, 3201-3210.

26 J. F. Mano, G. A. Silva, H. S. Azevedo, P. B. Malafaya, R. A. Sousa, S. S. Silva, L. F. Boesel, J. M. Oliveira, T. C. Santos, A. P. Marques, N. M. Neves and R. L. Reis, J. R. Soc., Interface, 2007, 4, 999-1030.

27 Y. Man, P. Wang, Y. Guo, L. Xiang, Y. Yang, Y. Qu, P. Gong and L. Deng, Biomaterials, 2012, 33, 8802-8811.

28 S. Ausländer, M. Wieland and M. Fussenegger, Metab. Eng., 2012, 14, 252-260.

29 G. D. Nicodemus and S. J. Bryant, Tissue Eng., Part B: Rev., 2008, 14, 149-165.

30 X. Bai, R. Fang, S. Zhang, X. Shi, Z. Wang, X. Chen, J. Yang, X. Hou, Y. Nie, Y. Li and W. Tian, J. Bioact. Compat. Polym., 2013, 28, 126-140.

31 S. Sakai, S. Yamaguchi, T. Takei and K. Kawakami, Biomacromolecules, 2008, 9, 2036-2041.

32 S. Manju, C. V. Muraleedharan, A. Rajeev, A. Jayakrishnan and R. Joseph, J. Biomed. Mater. Res., Part B, 2011, 98, 139149.

33 C. G. Gomez, M. Rinaudo and M. A. Villar, Carbohydr. Polym., 2007, 67, 296-304.

34 M. Friedman, J. Agric. Food Chem., 2004, 52, 385-406.

35 A. Vasconcelos, A. C. Gomes and A. Cavaco-Paulo, Acta Biomater., 2012, 8, 3049-3060.

36 T.-P. Nguyen and B.-T. Lee, J. Biomater. Appl., 2012, 27, 311321.

37 G. L. Peterson, Anal. Biochem., 1977, 83, 346-356.

38 B. Balakrishnan, S. Lesieur, D. Labarre and A. Jayakrishnan, Carbohydr. Res., 2005, 340, 1425-1429.

39 T. Painter and B. Larsen, Acta Chem. Scand., 1970, 24, 813833.

40 B. Smitha, S. Sridhar and A. A. Khan, Eur. Polym. J., 2005, 41, 1859-1866.

41 J. H. Kim, J. H. Kim, J. Jegal and K.-H. Lee, J. Membr. Sci., 2003, 213, 273-283.

42 H. Lee, S.-H. Ahn and G. H. Kim, Chem. Mater., 2012, 24, 881-891.

43 H.-A. Kang, M. S. Shin and J.-W. Yang, Polym. Bull., 2002, 47, 429-435.

44 T. Salomonsen, H. M. Jensen, F. H. Larsen, S. Steuernagel and S. B. Engelsen, Carbohydr. Res., 2009, 344, 2014-2022.

45 T. Salomonsen, H. M. Jensen, F. H. Larsen, S. Steuernagel and S. B. Engelsen, Food Hydrocolloids, 2009, 23, 15791586.

46 A. Saarai, T. Sedlacek, V. Kasparkova, T. Kitano and P. Saha, J. Appl. Polym. Sci., 2012, 126, E79-E88. 
47 J. Ye, J. Xiong and R. Sun, Carbohydr. Polym., 2012, 88, 14201424.

48 R. M. Issa, A. M. Khedr and H. Rizk, J. Chin. Chem. Soc., 2008, 55, 875-884.

49 G. Yang, L. Zhang, T. Peng and W. Zhong, J. Membr. Sci., 2000, 175, 53-60.

50 C. Peña, K. de la Caba, A. Eceiza, R. Ruseckaite and I. Mondragon, Bioresour. Technol., 2010, 101, 6836-6842.

51 L. Vervoort, I. Vinckier, P. Moldenaers, G. Van den Mooter, P. Augustijns and R. Kinget, J. Pharm. Sci., 1999, 88, 209214.
52 J. P. Soares, J. E. Santos, G. O. Chierice and E. T. G. Cavalheiro, Ecl. Quim. Sao Paulo, 2004, 29, 53-56.

53 A. Pawlicka, A. C. Sabadini, E. Raphael and D. C. Dragunski, Mol. Cryst. Liq. Cryst., 2008, 485, 804-816.

54 J. F. Martucci, A. Vázquez and R. A. Ruseckaite, J. Therm. Anal. Calorim., 2007, 89, 117-122.

55 K. Cai, J. Zhang, L. Deng, L. Yang, Y. Hu, C. Chen, L. Xue and L. Wang, Adv. Eng. Mater., 2007, 9, 1082-1088.

56 E. Rosellini, C. Cristallini, N. Barbani, G. Vozzi and P. Giusti, J. Biomed. Mater. Res., Part A, 2009, 91, 447-453.

57 S.-C. Wu, W.-H. Chang, G.-C. Dong, K.-Y. Chen, Y.-S. Chen and C.-H. Yao, J. Bioact. Compat. Polym., 2011, 26, 565-577. 